\title{
Experimental Validation of ADACOR Holonic Control System
}

\author{
Paulo Leitão ${ }^{1}$ and Francisco Restivo ${ }^{2}$ \\ 1 Polytechnic Institute of Bragança, Quinta Santa Apolónia, Apartado 1134, \\ P-5301-857 Bragança, Portugal \\ pleitao@ipb.pt \\ 2 Faculty of Engineering, University of Porto, Rua Dr. Roberto Frias, \\ P-4200-465 Porto, Portugal \\ fjr@fe.up.pt
}

\begin{abstract}
In the last years, several manufacturing control architectures using emergent paradigms and technologies, such as multi-agent and holonic manufacturing systems, have been proposed to address the challenge of developing control systems capable of handling certain types of disturbances at the factory level. One of these holonic architectures is ADACOR, which integrates a set of paradigms and technologies for distributed manufacturing systems complemented by formal modelling techniques, to achieve a flexible and adaptive holonic/collaborative control architecture. The results obtained in the first experiments using the ADACOR architecture are presented in this paper, and also compared to the results produced by other control architectures. For this purpose a set of quantitative and qualitative parameters were measured, to evaluate static and dynamic performance of the control architectures.
\end{abstract}

\section{Introduction}

In general, manufacturing systems are heterogeneous environments, comprising a variety of hardware and software applications. They are also asynchronous, stochastic and dynamic environments, with certain resources becoming unavailable and additional resources being introduced at random times, new jobs arriving continuously to the system, new products being frequently defined, and new regulations, such as quality and safety specifications, being regularly announced.

The economical and technological trends, associated to the customer satisfaction and products shorter life cycle impose new requirements to manufacturing systems that lead to new organizational structures (distributed, dynamic and open), flexibility and agility to support volatile and dynamic markets.

To face these requirements the new generation of manufacturing control systems should exhibit important features, such as agility (reacting rapidly to the occurrence of disturbances), re-configurability (changing dynamically its configuration, without stopping or re-starting the process), scalability (accepting the addition of new components, without the need to re-design, re-program or re-initialize the existing ones), re-usability (allowing to re-use past or previous 
solutions to simplify development) and intelligence (anticipating future demands and learning from the past experience).

In the last years, several manufacturing control architectures using emergent paradigms and technologies, such as multi-agent and holonic manufacturing systems, have been proposed to address this challenge (see [1234]). One of these holonic architectures is ADACOR (ADAptive holonic COntrol aRchitecture for Distributed Manufacturing Systems) [5], which integrates a set of paradigms and technologies for distributed manufacturing systems (HMS, MAS, ...) complemented by formal modelling techniques (Petri nets, AUML, ...), to achieve a flexible and adaptive holonic/collaborative control architecture.

ADACOR holonic control system is built upon a set of autonomous, cooperative and self-organized holons, each one representing a manufacturing component. ADACOR defines four holon classes [5]: product, task, operational and supervisor. The product holons represent the products available in the factory catalogue, the task holons represent the production orders launched to the shop floor and the operational holons represent the physical resources available in the shop floor. The supervisor holons provide co-ordination and optimization services to the holons under their supervision.

The adaptive ADACOR production control approach is neither completely decentralized nor hierarchical, but balances between a more centralized and a more flat approach, passing through other intermediate forms of control [5]. The presence of supervisor holons in a decentralised system, and the presence of selforganization capability associated to each ADACOR holon allows the evolution or the re-configurability of the control system, combining the global production optimization with the agile reaction to unpredictable disturbances.

ADACOR production control evolves in time between two alternative states, stationary and transient states [5]. In stationary state the holons are organized in a hierarchical-like structure, with supervisor holons coordinating several operational and/or supervisor holons. The role of each supervisor holon is to introduce global optimization in the production process. The transient state, triggered with the occurrence of disturbances, is characterized by the re-organization of the holons in a heterarchical-like control architecture, allowing the agile reaction to disturbances. This re-organization is performed through the self-organization of each holon, mapped with the increase of its autonomy and the propagation of the disturbance to the neighbor holons using ant-based techniques. After the disturbance recovery, the operational holons reduce their autonomy, evolving the system to a new control structure (often returning to the original one).

The validation of ADACOR concepts requires their implementation in a real environment, to analyze their correctness and applicability. In spite of the promising perspectives of the holonic manufacturing paradigm and the research developed by the holonic community, only few industrial implementations were reported in the literature, such as those described in [67]. This paper describes the experimental validation of ADACOR concepts in a flexible manufacturing system. The experimental results extracted from the implementation and testing allows to evaluate the ADACOR control system performance, both in terms of 
quantitative indicators directly related to production parameters and of qualitative indicators related to the dynamical behaviour of the system.

\section{Experimental Case Study}

A pilot installation has been used to validate the ADACOR holonic control system, aiming to address two main objectives: i) validate the concepts and the implementation, i.e. to verify if the system works as it was specified, and ii) evaluate the performance to conclude about the merits of the proposed concepts. The case study used in this work will be described in the next sections.

\subsection{Pilot Installation}

The pilot installation is a semi-virtual laboratorial platform based on the flexible manufacturing system of the CIM Centre of Porto, which is 'extended' for our purpose with two virtual manufacturing cells, to provide the necessary hardware/software redundancy and flexibility in accommodating alternative solutions at the production planning level, as illustrated in Figure 1, The flexible manufacturing platform is organized as a set of four physical cells: manufacturing cell, assembly cell, storage and transportation cell and maintenance and setup cell. Cells $\mathrm{B}$ and $\mathrm{C}$ in the figure do not exist in the real platform.

The assembly cell is responsible for the assembly of the components to achieve the final products. This cell has a SCARA robot Adept Three from Adept Technology. Coupled to the robot, there is a CCD camera from PULNIX, associated to an artificial vision system Cognex 4200EX from Cognex Corporation.

The storage and transportation cell is responsible for the transportation of materials within the shop floor and for the temporary storage of materials.

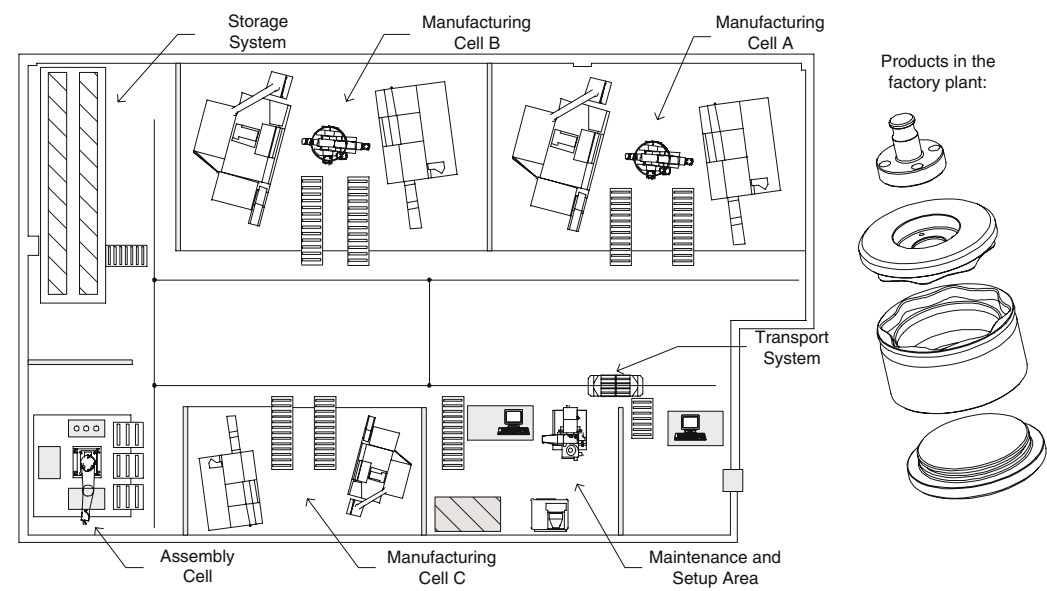

Fig. 1. Plant layout of the case study production system 
This cell has an AGV (Automatic Guided Vehicle) and an Automated Storage/Retrieval System. The presence of the AGV allows variable routing of the products flow.

The maintenance and setup cell is responsible for maintenance, setup and recovery operations, assembly of tools, calibration of tools and grippers, and palletizing and demagnetizing of the materials that circulate in the shop floor. This cell includes a tool calibration system AR2000GA from Elbo Controlli, a palletising table and several equipments to support the maintenance operations.

The manufacturing cell has two $\mathrm{CNC}$ machines and one anthropomorphic robot for the load/unload of these machines. One of these machines is a turning center Lealde TCN10, with a SIEMENS Sinumerik 880T controller. The other machine is a milling center Kondia B500, with a FANUC 16MA numerical control. The robot is a KUKA IR163/30.1 with a SIEMENS RC3051 controller.

The production system contains other types of resources, namely buffers and containers. Each machine has its input/output buffer, to de-couple it from the transport system. The containers bring the material to be processed in the machine or the cell and take away the pieces produced.

In this pilot plant, four different (sub-)products are produced, as illustrated also in Figure 1, named Base, Body, Cover and Handle. When assembled, they can create two different final products: Box and Ashtray. The Ashtray product comprises the assembly of the Base and the Body sub-products, and the Box product comprises the assembly of all designed sub-products.

\subsection{Manufacturing Scenarios}

The experiment considers three different plant scenarios: i) the first plant scenario considers that no unexpected disturbance will occur, ii) the second plant scenario considers the occurrence of failures in one turning machine (cell B), with a probability of $25 \%$, and that, in case of failure, the part is destroyed and the machine is down during 60 seconds for the recovery procedures, and iii) the third plant scenario considers the occurrence of failures in the turning machines of cells $\mathrm{B}$ and $\mathrm{C}$, with the same disturbance model of the previous scenario.

In this experimental test it is considered that no setups are executed, since machines are equipped with the required tools to execute a range of operations. The transport operations are performed by a single AGV and orders are queued by order of arrival. The execution of each transport operation takes 5 seconds.

Each individual book of orders comprises the production of 6 production orders: 2 bodies, 2 bases, 1 handle and 1 cover. The experimental test reported in this paper considers a plant load of three books of orders, i.e. 18 production orders (involving 51 operations). All the production orders belonging to the same book of orders arrive to the production system at the same time, but different books of orders arrive sequentially to the production system.

Each experiment was executed 6 times, and average values and standard deviations were computed. As the control systems run on a multitask platform with parallel threads and inter-process communications, originating a certain degree of randomness in the allocation of operations, and thus producing some 
stochastic variations in the processing times, more accurate results are obtained this way.

\subsection{Performance Indicators}

Performance indicators can be classified as qualitative or quantitative. The quantitative indicators are based on different production performance measures, such as the lead time and throughput. The qualitative indicators are of a more subjective nature and reflect properties of the manufacturing control solution, such as the agility and flexibility, which cannot be directly obtained from the production data. In this experimental test, the ADACOR holonic control system was evaluated by analyzing the following performance indicators: manufacturing lead time, throughput, repeatability, resource utilization and agility.

The manufacturing lead time is the total time required to process a given product through the factory plant, and comprises the setup time, the no-operation time, the idle time and the processing time. The shorter the lead time is, more products can be produced by the production plant in the same period of time. The lead time reflects factory plant optimization level and productivity.

The throughput is an indicator of the productivity of a manufacturing system, and is defined here as the number of items produced per time unit. In the context of this work, the throughput was measured as the ratio between the number of parts produced in the experience and the batch time.

The resource utilization is defined as the percentage of processing time during a time interval. The average resource utilization is equal to the mean value of the percentage of utilization of all resources in the system. The analysis of the standard deviation of the utilization of all the resources allows to verify if the manufacturing load is evenly distributed by all the resources or concentrated in a few ones. A high value for this parameter may indicate the existence of overloaded resources, and the need to re-allocate some load to other resources.

The repeatability of the manufacturing control system is given by the mean value of the standard deviation of the percentage of utilization of all resources of the system over the several experiences. The smaller the repeatability is, more repeatable is the manufacturing control system production plan.

The agility of a control system can be defined as the capability to react in a short period of time to the occurrence of unexpected disturbances, more exactly, the time needed by the system to recover properly from the occurrence of a disturbance.

In this experimental test, the agility parameter is evaluated by running $n$ experimental tests and analyzing the loss of productivity in presence of disturbance scenarios. For that purpose, it is necessary to know in first place the time required to produce a specific amount of items with no disturbances. Then, it is measured the time required to execute the same products, under a disturbance scenario. Having these two values, it is possible to calculate the throughput in each case, and the percentage of reduction of throughput, which is the loss of 
productivity. The loss of productivity reflects indirectly how agile the system is. The smaller the loss of productivity value is the higher the agility of the system will be.

\section{Prototype Implementation and Operation}

The ADACOR control system prototype was implemented using multi-agent systems technology [8]. From the set of available commercial and academic agent development platforms 9, it was chosen the JADE (Java Agent Development Framework) platform (see http://jade.cselt.it/), which complies with the FIPA (Foundation of Intelligent Physical Agents) specifications.

\subsection{Implementation}

An ADACOR holon is a Java class that extends the Agent class provided by the JADE framework, inheriting basic functionalities, such as registration services, remote management and sending/receiving ACL messages, and adding features that represent the specific behaviour of each ADACOR holon class. The behaviour of each ADACOR holon uses multi-threaded programming, over the concept of JADE's behaviour, to allow the execution of several actions in parallel. The set of behaviours (each one corresponding to a kind of thread) launched at the start-up and those that can be invoked afterwards are provided in the form of Java classes.

The communication between distributed holons is done over the Ethernet network, the messages being encoded using the FIPA-ACL communication language. The content of the messages is formatted according to the FIPA-SL0 language and the meaning of the message content is standardized according to the ontology defined by the ADACOR architecture.

The decision component of each ADACOR holon uses declarative and procedural approaches to represent knowledge and to regulate the holons behaviour 8]. The central element in the decision component is the rule-based system developed using JESS (Java Expert System Shell), which applies declarative knowledge expressed in a set of rules. ADACOR holons uses also procedural knowledge embodied in procedures that are triggered as actions by some rules, each one being responsible for the execution of a particular set of actions. The scheduling algorithm is an example of this type of knowledge representation.

The implementation of operational holons that represent physical automation resources requires the development of wrapper interfaces, supporting the integration of those resources within the holon. ADACOR introduces the virtual resource concept making transparent the intra-holon interaction [8]. The development of a virtual resource for each manufacturing device encompasses the implementation of the services at the server side (the real automation resource), which will be invoked on the client side (the logical part of the operational holon). The client ignores the details of this implementation and each virtual resource can be re-used by other similar resources or holonic control applications. 


\subsection{Operation}

A Factory Plant Supervisor tool was developed to monitor, in an integrated way, the production activities in the factory plant. This tool, represented in Figure 2, allows visualizing the state of the manufacturing resources present in the factory plant. The graphical animation associated to the AGV helps to understand the material flow in the factory plant.

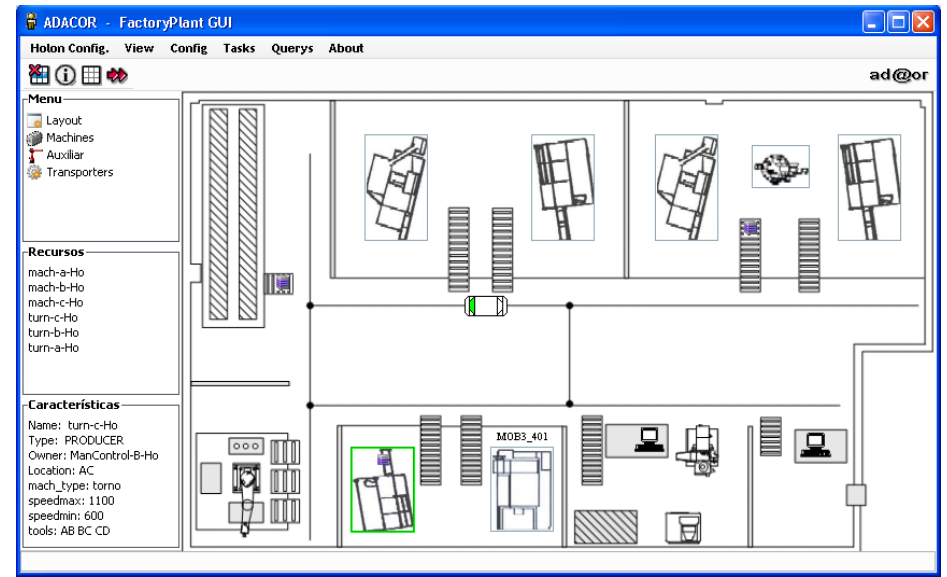

Fig. 2. Screenshot of ADACOR Factory Plant Supervisor

During the experimental test the several holons presented in the system were distributed by different PCs, running in different platforms such as Windows XP, Windows 2000 and Linux. This allowed demonstrating that ADACOR control system supports the heterogeneity presented in industrial automation scenarios.

The experience gained during the prototype implementation, debugging and testing allows extracting some conclusions about the operation of ADACOR holonic control system. In a first instance, it was verified that it works as specified, either in normal operation or in presence of disturbances. This was one of the major objectives of the experimental validation.

Additionally, the re-configurability of the ADACOR holonic control system was proven, since the system reacted correctly to the introduction, removal and modification of manufacturing components. Specially, it was shown that when a supervisor or operational holon breaks down or leaves the system, the other holons continue their way finding alternative solutions to execute the production plan. This is mainly supported by the plug-and-produce characteristic associated to the ADACOR holons, i.e. each ADACOR holon works autonomously, not requiring the need for additional re-design, re-program and re-start of other components. 


\section{Analysis of Quantitative Performance Indicators}

The first set of experimental tests evaluates the behaviour of the ADACOR control approach, by comparing its performance with the hierarchical and heterarchical control approaches, focusing in the analysis of the quantitative indicators.

An important remark is related to clarify that all the three control approaches uses the same prototype platform: i) in the hierarchical control approach, the holons are organized in a hierarchical control structure, using supervisor holons, ii) in the heterarchical control approach, the holons run on a completely decentralized control structure, without the presence of supervisor holons, and iii) in the ADACOR holonic control approach, the holons are organized in a hierarchical control structure, using supervisor holons and enabling the self-organization of each operational holon to support the agile re-organization of the control structure in case of emergency.

\subsection{Stable Scenario}

In a scenario without the presence of unexpected disturbances, the system operates in a predictable way. The results of this experimental test are summarized in the Figure 3. In a stable scenario the holons of the ADACOR control approach are organized in a hierarchical structure, presenting the same behaviour as in the hierarchical-like control, therefore showing the same experimental values.

In stable scenarios the hierarchical-like and ADACOR control approaches present smaller values of manufacturing lead time $(336,2)$ and higher values of the throughput $(49,4)$ than the heterarchical-like control approach (respectively $387,2$ and 46,0$)$. The better performance presented by those approaches results from the better production planning achieved by the centralized entities, i.e. a supervisor holon that elaborates optimized production plans.

Analyzing the repeatability of the production planning, which is a measure of its predictability, it is clear that the repeatability in hierarchical-like and ADACOR control approaches is better than in the heterarchical-like control approach. In fact, in the heterarchical-like control approach the global schedule is achieved by the interaction of operational holons that have a partial view of the entire system, making difficult to achieve the global optimization.

The type of production has also strong impact in the degree of production optimization achieved. It was verified that for operations with short processing
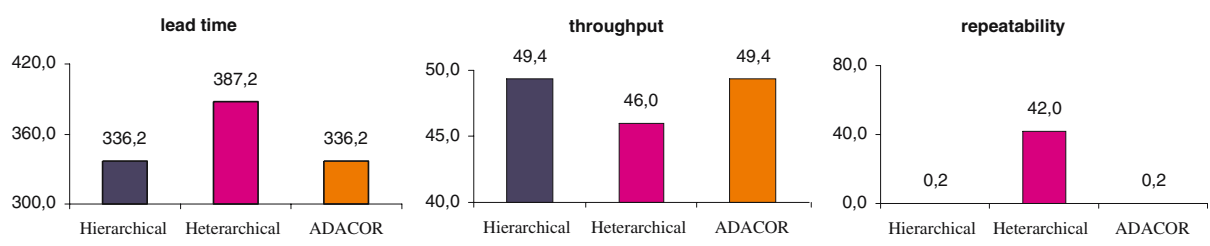

Fig. 3. Performance of evaluated control approaches for scenarios with no disturbance 
times, the better performance resulting from the global optimization present in hierarchical-like and ADACOR control approaches is less visible, while in cases of operations with longer processing times, the non-optimised schedules present in heterarchical-like control approaches lead to even worse performance. This is due to the fact that longer processing times are more sensitive to weak global optimization.

\subsection{Disturbance Scenarios}

The second experimental test considers the occurrence of unexpected disturbances in the turning machine of cell $\mathrm{B}$, according to the probabilistic disturbance model described in section 2.2. The results obtained in this experimental test are summarized in the Figure 4.

The first conclusion extracted from these experimental results is the degradation of all performance indicators in the presence of disturbances.

Analysing the lead time and throughput parameters, it is possible to verify that the hierarchical-like control approach still presents better performance than the heterarchical-like control approach. However, it is possible to verify that the difference of performance between hierarchical-like and heterarchicallike control approaches has been significantly reduced, specially in terms of the throughput parameter (reduction of $8,7 \%$ for the lead time and $55,9 \%$ for the throughput).

The occurrence of disturbances increases the entropy and unpredictability of the control system. It was verified that in disturbance scenarios the differences between the predictability exhibited by the several evaluated control approaches are smaller (i.e. between 52,9 to 66,9 ). Thus, the proposed ADACOR holonic control approach presents promising performance results, since it shows better response to the disturbance scenario, illustrated by smaller value of manufacturing lead time $(337,7)$ and higher value of throughput $(46,6)$, than the hierarchical-like and heterarchical-like control approaches.

The second disturbance model was used to compare the response of the three control approaches to the different levels of entropy caused by the occurrence of disturbances. In this scenario, it is assumed that failures can occur in turning machines of cells B and C. Figure 5 illustrates the results obtained during the execution of this experimental test.
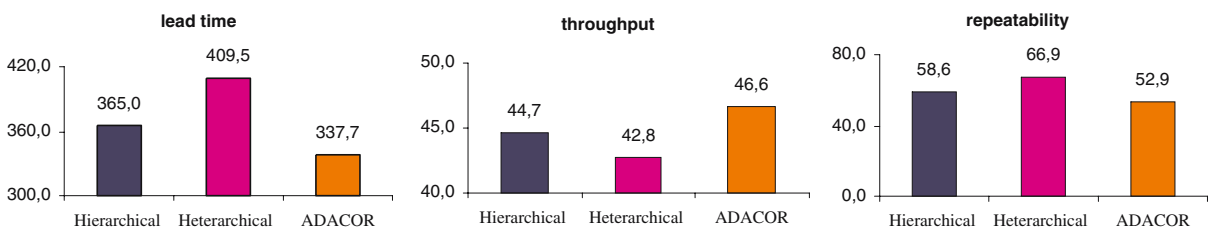

Fig. 4. Performance of evaluated control approaches for disturbance scenarios 

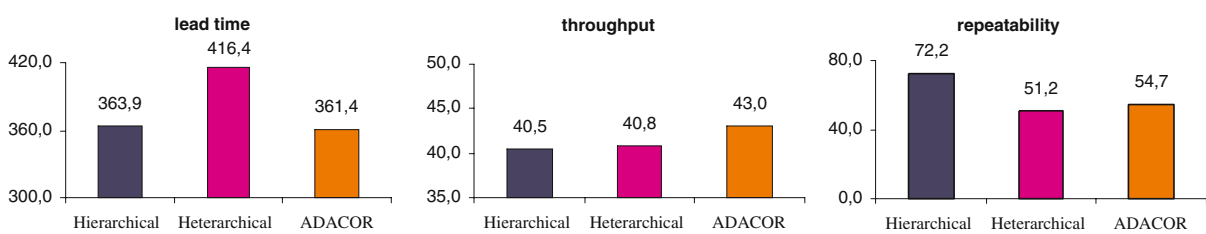

Fig. 5. Performance of evaluated control approaches for the 2nd disturbance model

The experimental results confirm the observations done during the previous experimental test, being clear that the performance of each control approach suffers with the increase of entropy associated to the disturbance model.

\subsection{Analysis of Resource Utilization}

For the purpose of the resource utilization analysis, only the three turning machines were considered, since they are the ones that provide alternative paths to execute the operations to manufacture the package of available products. The average and the standard deviation of the percentage of resource utilization, for the stable and first disturbance scenarios are summarized in the Figure 6.

Analyzing the experimental results, it is possible to verify that the hierarchicallike and ADACOR control approaches present higher percentage of resource utilization than the heterarchical-like control approach, either in the stable and disturbance scenarios, which demonstrates their better production plan optimization. It can also be observed that the percentage of resource utilization is higher in disturbance scenarios than in the stable scenario, due to the execution of additional work orders launched to the shop floor, after the occurrence of machine failures that destroyed the part.

The analysis of the standard deviation of the percentage of resource utilization, which gives an idea about how the planning and control system distributes the load by the available resources, allows verifying that the heterarchical-like control approach presents the worse behaviour in the load distribution variability, as expected. On the other hand, the ADACOR control approach presents values for the standard deviation that are even smaller than those for the hierarchicallike control approach.

mean of percentage of resource utilization

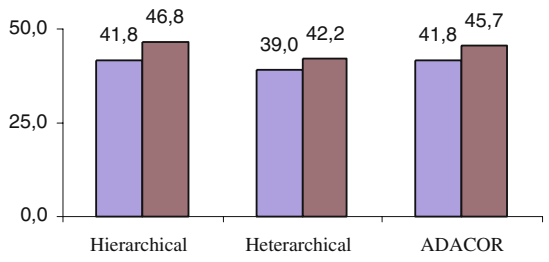

standard deviation of percentage of resource utilization

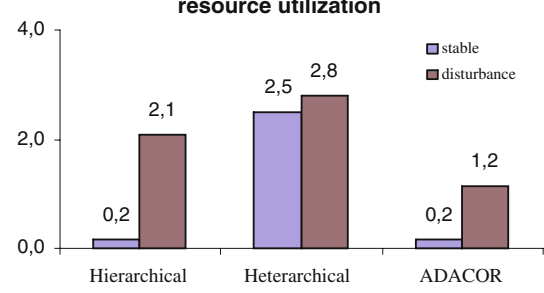

Fig. 6. Experimental results of the resource utilization 


\section{Analysis of Qualitative Performance Indicators}

In this section, the ADACOR control system is evaluated by analyzing a single qualitative performance parameter, the agility. The comparison of the loss of productivity values for the two disturbance models is illustrated in Figure 7.

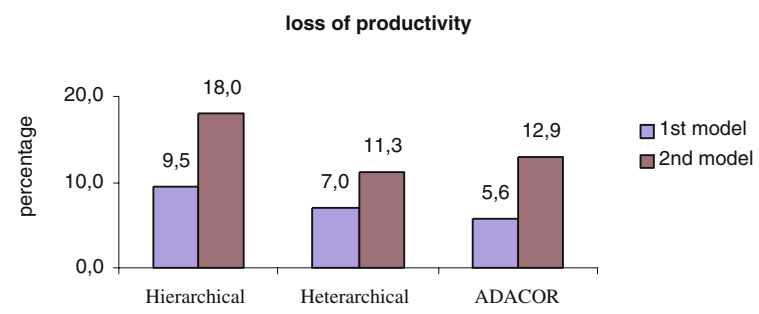

Fig. 7. Loss of productivity of the evaluated control approaches

It is possible to verify that the ADACOR control approach presents similar values to those exhibited by heterarchical-like control approach. As expected, the hierarchical-like control approach presents the higher loss of productivity. As the agility is inversely proportional to the loss of productivity, the experimental results show that the ADACOR control approach exhibits the same levels of agility of those of the heterarchical-like control approach. In scenarios where disturbances are more frequent, the levels of agility presented by the several control approaches are reduced.

Analyzing simultaneously the agility and the manufacturing lead time, the results obtained in these experimental tests reveals that the ADACOR holonic control system presents promising performance results, since it shows better response to the disturbance scenario, illustrated by smaller value of manufacturing lead time, and confirm that the ADACOR holonic control system combines the hierarchical and heterarchical best features, presenting similar values of agility to the heterarchical approach, but better production optimization.

\section{Conclusions}

ADACOR architecture addresses the agile reaction to the occurrence of unexpected disturbances at shop floor level, by introducing an adaptive production control approach, that evolves dynamically through different control structures supported by the self-organization capability associated to each ADACOR holon.

The results obtained in the first experiments using the ADACOR architecture were presented, and compared to the results produced by other control architectures. Three scenarios were devised, with different levels of occurrence of failures, using the same book of orders. Mean value and standard deviation of a set of quantitative and qualitative parameters were measured, to evaluate static and dynamic performance of the control architectures. 
It was possible to conclude first of all that ADACOR architecture was able to handle all the possible combinations of situations, allowing concluding that the ADACOR concepts are sound and ready to be used in real situations.

Performance measures also showed that ADACOR control architecture exhibits the better performance in what concerns to dynamical behaviour of the production facility after the occurrence of failures.

An important issue to consider in future work within ADACOR is to focus in the performance measurement of manufacturing control systems. Some ongoing work in this issue is being done by Intelligent Manufacturing Systems Network of Excellence (IMS-NoE), Special Interest Group on Benchmarking and Performance Measures of on-line Production Scheduling Systems (see http://www.imsnoe.org), where the authors are associate members.

\section{References}

1. Van Brussel, H., Wyns, J., Valckenaers, P., Bongaerts, L. and Peeters, P.: Reference Architecture for Holonic Manufacturing Systems: PROSA. In: Computers In Industry, 37, 1998, pp. 255-274.

2. Deen, S.M. (ed.): Agent-Based Manufacturing: Advances in the Holonic Approach, Springer Verlag Berlin Heidelberg, 2003.

3. Maturana, F. and Norrie, D.: Multi-Agent Mediator Architecture for Distributed Manufacturing. In: Journal of Intelligent Manufacturing, vol. 7, 1996, pp.257-270.

4. Chirn, J.-L. and McFarlane, D.: A Holonic Component-Based Approach to Reconfigurable Manufacturing Control Architecture. In: Proc. of the Intern. Workshop on Industrial Applications of Holonic and Multi-Agent Systems, 2000, pp. 219-223.

5. Leitão, P., Colombo, A.W. and Restivo, F.: ADACOR, A Collaborative Production Automation and Control Architecture. In: IEEE Intelligent Systems, 20(1), 2005, pp. $58-61$.

6. Colombo, A. W., Schoop, R. and Neubert, R.: Collaborative (Agent-Based) Factory Automation. In: The Industrial Information Technology Handbook, R. Zurawski (ed), CRC Press, 2004.

7. Maturana, F., Staron R., Tichy, P. and Slechta, P.: Using Dynamically Created Decision-Making Organisation (Holarchies) to Plan, Commit and Execute Control Tasks in a Chilled Water System. In: Proceedings of the 3rd Int'l Workshop on Industrial Applications of Holonic and Multi-Agent Systems, 2002, pp. 613-619.

8. Leitão, P., Casais, F. and Restivo, F.: Holonic Manufacturing Control: A Practical Implementation. In: Emerging Solutions for Future Manufacturing Systems, L. Camarinha-Matos (ed.), Springer Science+Business Media, 2004, pp. 33-44.

9. Vrba, P.: JAVA-Based Agent Platforms Evaluation. In: Holonic and Multi-Agent Systems for Manufacturing, LNAI 2744, Springer-Verlag, 2003, pp. 47-58. 